\section{Overuse of antibiotics as a key driver to antibiotic resistance in Morocco: A short review with potential solutions}

\section{Abstract}

Antimicrobial resistance is a global public health challenge, which has accelerated by the overuse of antibiotics worldwide. Increased antimicrobial resistance is causing severe infections, complications, longer hospital stays and increased mortality. Steps must be taken to reverse the damage that has already been done and prevent further resistance from developing. This review study will examine the context of antimicrobial resistance in general with emphasis on its presence in Morocco. Second, the social situation that led to antibiotic overuse and misuse seen in both health care professionals and the public will be discussed. Third, offer solutions that have been suggested to prevent and reverse this problem.

Keywords

Antibiotic Resistance; Overuse of Antibiotics; Morocco.
Amine Ousaid ${ }^{1}$, Jaouad Akrim", Youssef Khayati ${ }^{1}$

\section{Laboratory of Drug Sciences, Biomedical and Biotechnological Research, Faculty of Medicine and Pharmacy, Hassan II University. Casablanca. Morocco.}

Contact information:

Amine Ousaid.

झ ousaid237482@gmail.com

Received 06-04-2020; Accepted 06-05-2020

\title{
Introduction
}

The rapid emergence of resistant bacteria is occurring worldwide including in Morocco and its neighboring countries endangering the efficacy of antibiotics, which have transformed medicine and saved millions of lives [1, 2]. The antibiotic resistance crisis has been attributed to the overuse and misuse of these medications. In addition, the lack of new drug development by the pharmaceutical industry due to reduced economic incentives and challenging regulatory requirements is a serious problem $[3,4]$.

Many physicians and other health care professionals prescribe antibiotics with little concern of its overuse. It has become practice to prescribe antibiotics for common and minor sore throat, which often 
caused by viruses and would be normally eliminated by a healthy immune system. Antibiotic overuse is leading factor to drug resistance in bacteria, and will ultimately result in losing most of the currently available antimicrobial medications $[5,6]$.

The aim of this study is to present data on antibiotic resistance in Morocco and propose several solutions to this growing issue.

\section{Brief history of antibiotic resistance}

Penicillin, the first antibiotic discovered by Alexander Fleming in 1928 by fortunate accident was believed to be a miracle drug, saving the lives of thousands of soldiers in World War II and improving the quality of life for the public. However, shortly thereafter, penicillin resistance became a substantial clinical problem [7]. In response, new beta-lactam antibiotics were discovered, developed and deployed for medical use. The first case of methicillin-resistant Staphylococcus aureus (MRSA) was identified in the United Kingdom in 1962. Vancomycin was used in 1972 for the treatment of methicillin-resistance in both $S$. aureus and coagulase-negative staphylococci. In 1979, first case of vancomycin resistance were reported in coagulase-negative staphylococci. Antibiotic development continued and increased throughout the following decades where numerous new antibiotics are becoming available for medical use; including carbapenems, cephalosporins, tetracyclines, aminoglycosides, monobactam, macrolides, streptogramins $[1,8]$.

The plethora of antibiotics now available are widely and readily prescribed by medical providers for human and animal. All are being used in an alarmingly pattern that is fueling and contributing to the current antibiotic resistance problem. In short, this crisis is a result of many factors; pharmaceutical companies practice in offering incentives for physicians, lack of solid knowledge about antibiotics of prescribers and antibiotic resistance pattern in their society, patients negligees, misuse and overuse in agriculture and animal husbandry, and the genetic abilities of many bacteria species to develop rapidly resistance to certain antibiotics $[8,9]$.

\section{Context Leading to Antibiotic Resistance}

\section{Role of pharmaceutical industry}

Pharmaceutical companies discover, develop, and market new antibacterials. Clearly, it is in the best interests of pharmaceutical companies to promote wide use of antibacterials in order to justify research and development costs. Big pharmaceutical companies obtained huge profits and ensured the companies' commitment to develop and market many new antibiotics for medicine. This fact, coupled with the willingness of some physicians to prescribe the latest antibacterial, has undoubtedly increased the frequency of resistance over time. Between 1988 and 2000, many new antibiotics were available and profitable during these years [11].

Animal testing laws in particular were in the past much easier to carry out testing [12]. Since 2003, only one new antibiotic per year on average has been approved by the FDA for medical treatment [11]. The largest names in pharmaceuticals, including Wyeth, Eli Lilly, Procter and Gamble, Roche, Abbott Laboratories, and Aventis have either largely reduced their antibiotic research and development or terminated their antibiotic research entirely [10-11]. It is simply not as profitable to focus on antibiotics as other areas of drug research. These companies have shifted their research and development focus to medications with wider markets and higher profit margins. Drugs that are used in the treatment of chronic diseases are of particular interest to big pharmaceutical companies. Antibiotics are much more difficult and expensive to manufacture than medications for conditions such as diabetes, high cholesterol, depression and arthritis. Antibiotics are far less profitable than other so called "maintenance medications". Antibiotics are only used for a short 
duration and will only be utilized periodically during a person's lifetime. It is understandably difficult to make any profit on the development of a medication that will only be used for approximately ten days at a time every other year, especially when antibiotics are so difficult to develop and test for human use. There is a lot more money to be made from so called "blockbuster" drugs than from antibiotics that will only be used periodically and for a short duration by patients [11].

A study published by Huttner et al. [13] found that pharmaceutical companies spent roughly 1.6 billion dollars in 1998 to promote antibiotics to customers, hospitals, doctor's offices, and pharmacies. This investment could easily be spent in the pursuit of new antibiotics or on safe antibiotic usage campaigns. Sadly, it is being used only to market and encourage the further use of already well-established antibiotics.

\section{Role of medical doctors}

Physician's decisions with regard to prescriptions are very complex and determined by many factors. Clearly, in cases of infectious disease, the decision to treat is based on the diagnosis of bacterial infection or on the likelihood of a bacterial infection. The dose and type of antibacterial are chosen on the basis of the likely pathogen and its local in vitro susceptibility, the severity of the infection, and the condition of the patient. In most situations, neither the pathogen nor its in vitro susceptibility is known. Antibiotic use is increasing substantially worldwide, though it is increasing to the largest degree in the United States and other highly developed countries $[1-2,5]$. Antibiotic over-prescription is understood to be very expensive and known to contribute to the growing resistance of bacteria [6-8].

Antibiotics are being vastly overused and misused and are often prescribed for respiratory viral illnesses that will not be covered by antibiotic medication. Prescribing antibiotics for self-limiting illnesses is perhaps the main reason for the emergence of antibiotic-resistant bacteria in the community setting [6-8]. It is perplexing to consider the reasons physicians, who are typically very highly educated individuals, choose to prescribe medications that will do more harm than good for the patient and society as a whole in the long run. This frightening trend has its roots in many factors. Despite their reasons, the truth remains that antibiotic prescription is not always based on clinical factors alone $[5,8]$.

It has been suggested that antibiotic over-prescribing can be correlated with the prescriber's individual personality and environment. Clinician characteristics that have been linked to unnecessary prescribing include years of practice, medical specialty, perceived patient expectations, and desire to please the patient [5-6]. General practitioners prescribe somewhere around ninety percent of the antibiotics prescribed in the United States for a given year and more than half of these are prescribed to treat respiratory tract infections [7]. Statistics are very close to these numbers for most highly developed European countries as well [13]. Urinary tract infections are the second most common disease for which antibiotics are typically prescribed. It is estimated that more than $50 \%$ of patients who present to a primary care facility with symptoms of a urinary tract infection are treated right away with antibiotics, despite the fact that urinary tract infections are incredibly self-limiting, and pose a very small threat to an individual's overall health and wellbeing [1, 14].

Treating urinary tract infections with antibiotics before performing the necessary culture and lab work is not only irresponsible from a microbial resistance point of view; it is also quite costly and ineffective in treating the illness. The patient may be given peace of mind knowing that he was prescribed something tangible, but the benefit will go no further. The harm done by prescribing antibiotics for urinary tractinfections far outweighs the good it will do to the patient. The majority of the responsibility for the ineffective and irresponsible prescribing of antibiotics lies with the prescribers, whether they 
be physicians, physician assistants, or other medical faculty [15]. However, the patient also bears a portion of the blame and responsibility. It is indeed patient expectations and presuppositions that make doctors feel bullied or forced into prescribing antibiotics either "just in case", or to please patients who will not be satisfied unless they leave with a prescription [8].

A study was performed in Iceland to examine how often and in what contexts medical practitioners; specifically family and general practitioners prescribe antibiotic drugs to patients who present with no clear or definite signs of bacterial infection. Arason calls this prescribing method "nonpharmacological prescriptions of antibiotics" [6]. The study found that the primary reasons doctors would prescribe antimicrobial drugs to a patient with a non-definite bacterial illness are all related to the ever changing doctor-patient relationship. In the region studied, the reason for this fluctuation in doctor-patient relations was supposed to be the lack of continuity in medical care that most patients sought out. Pressure from patients in a stressful society, the physician's heavy work pressure, the prescriber's own personality, any supplemental income or prescribing incentives they may be receiving from pharmaceutical companies, and the physician's lack of confidence or uncertainty in their diagnostic abilities were the main factors that resulted in a situation in which antibiotic prescriptions were prescribed as a kind of coping strategy for an uncomfortable situation with a patient [6]. A European study surveyed one thousand general practitioners about their prescribing habits and practices, and $55 \%$ of the general practitioners admitted to feeling under intense pressure from patients to prescribe antibiotics, even if the practitioner insisted and explained to them that the antibiotic was unnecessary and would be ineffective. A total of $44 \%$ of the general practitioners further admitted to prescribing antibiotics to certain patients just to get them to leave the office [7].
The over-prescription of antibiotic drugs is particularly prevalent among pediatric patients. A study in the Scandanavian Journal of Primary Health Care found that children under the age of seven consumed nearly $20 \%$ of the total antibiotic medication sold, though they comprised only about $10 \%$ of the total family care office visits. Over half of these patients were prescribed antibiotics due to ear infections, which are rarely caused by bacterial than viral infection [6]. A correlation was found between the amount of antibiotics an individual child consumed and the risk of that patient being a carrier of antibiotic resistant microbes, specifically penicillin nonsusceptible pneumococci, in the weeks following the medical treatment [6].

Pediatric ear infections are some of the most common illnesses that prescribers an antibiotic to treat [10]. Upper respiratory tract infections are the leading cause of antibiotic overprescribing in the outpatient setting, both for pediatric and adult patients [13]. Another study done in Sweden found that after the implementation of more thorough diagnostic techniques, fewer patients were being diagnosed with bacterial upper respiratory infections. Shockingly, the proportion of patients who were prescribed an antibiotic did not decrease as may be expected. The proportion of patients who were prescribed an antibiotic actually increased over the time period studied [6]. This study shows definitively that clinical factors such as bacterial culture workups, are very low on the list of reasons a prescriber would prescribe antibiotic drugs to a patient. A cause that may be a contributor to unnecessary antibiotic prescribing is the fact that antibiotic over-prescription has been shown to increase the likelihood that patients will return to physician office [7]. Knowing that one can go into a doctor's office and leave with definitive treatment, regardless of whether or not that treatment is appropriate for one's individual case and condition, will increase the likelihood of the individual bringing their business back to that pro- 
vider. Patient loyalty is likely a contributing factor to the antibiotic crisis [7-8].

\section{Role of patients}

Patients have become far more demanding for antibiotic treatment. They often believe that if they leave a doctor's office without an antibiotic or other tangible and definitive treatment, they are being short managed or cheated in some way. Patients consider physcians as lazy and uncaring if they choose not to prescribe a medication for the individual's illness [14]. Misconceptions and presuppositions are commonly present within the public's belief as it pertains to antibiotics. A study done in Europe looked at what the public believes about antibiotics and their effectiveness. The study found that about $50 \%$ of the patients surveyed believed that antibiotics were fully effective in treating viruses, cold, and flu and should definitely be prescribed in those cases [7]. This is alarming, because none of those illnesses will be improved by antibiotics. Antibiotics are effective only against bacterial infections, not against viruses like the ones that can cause ear infections, respiratory infections, flu, and the common cold.

Patient characteristics that coincide with excessive and unnecessary antibiotic prescribing include misperceptions about antibiotic effectiveness for viral illnesses, desire for a tangible product from the clinical encounter, age, ethnic consideration and insurance status [5, 7]. Older patients from underprivileged areas and minority patients were the groups that received unnecessary antibiotics the most. A study of Ackerman suggests that it is difficult to converse with some patients and explain them unpleasant or complicated information about usage of antibiotics [5]. These relation do make for a sometimes difficult interaction between physician and patients. Social characteristics of the doctor-patient relationship as mentioned above must definitely be considered when looking at the issue of antibiotic over-usage [5]. However, the ongoing cultural shift in the expected role of the patient from passive receiver of medical expertise to active medical "consumer" must also be heavily considered [7]. People expect to be sold something and, when they are turned away without medication, they feel cheated or short treated in some way.

\section{Role of bacterial culture}

The uncertainty involved in diagnostic culture testing also contributes to overprescribing of antibiotics, particularly in the primary care setting. A Dutch study done in 2005 showed that the use of antibiotics was very strongly linked to physicians wanting to avoid uncertainty in diagnosing patients rather than actual clinical indications of a bacterial illness [8]. Physicians want to eliminate the possibility of withholding antibiotic treatment from a patient only to have them suddenly develop a serious bacterial infection, and become severely ill or even die. The legal issues and moral questions weigh heavily on the backs of doctors facing these choices. The problem is that the likelihood of misdiagnosing a life threatening bacterial infection as a self-limiting viral illness is incredibly small. By providing a "blanket coverage" antibiotic prescription just to ease the patient's worries or prevent a second visit for the physician. The crisis of antibiotic resistance is being further propagated, and it is not beneficial to the patient or physician to practice antibiotic prescription for just please the patient's desire $[8,11]$.

\section{Antibiotic resistance in Morocco and neighboring countries}

Antibiotic resistance is rising to dangerously high levels in all parts of the world including Morocco and other neighboring African countries, and bacteria are becoming alarmingly antibiotic multidrug resistant. For example, Staphylococcus aureus is one the organism which has developed remarkable high level of resistance against multiple antibiotics, and complicating treatment. Historically, S. aureus resistance emerged within 2 years of the introduction of penicillin [17]. In 1942, the first penicillin-resistant $S$. aureus strain was detected, the semisynthe- 
tic antibiotic methicillin was then developed in the late 1950s developed as a type of a penicillinaseresistant penicillin, and methicillin-resistant $S$. aureus (MRSA) was clinically first identified in 1960 [17]. Infections due to MRSA strains are associated with higher mortality rates than infections caused by methicillin-susceptible strains. In addition, MRSA infection is associated with increased lengths of hospital stays and high health care costs [17].

Currently, over $95 \%$ of $S$. aureus is resistant to not only penicillin, but also ampicillin and the antipseudomonal penicillins [18]. Hospitals are breeding grounds for bacterial resistance because they provide an ideal environment for the development of multidrug resistant bacteria by promoting the overgrowth of particular strains of bacteria that carry the gene for drug resistance. Clostridium difficile is another example of this phenomenon. When antibiotics are used unnecessarily or indiscriminately, the normal bacteria that live in the gut can be disrupted and killed, allowing drug-resistant bacteria to overgrowth other types of bacteria. C. difficile is the most prominent of these bacteria. Antibiotic treatment of hospitalized patients causes alterations of the intestinal microbial composition, increased C. difficile colonization and consecutive toxin production leading to disruption of the colonic epithelial cells. Clinical symptoms of $C$. difficile infection range from mild diarrhea to potentially life-threatening conditions like pseudomembranous colitis or toxic megacolon [19]. Extensive antimicrobial treatment applies selective pressure on normal body bacteria. This pressure favors the emergence of certain strains of the bacteria that will be multidrug resistant to the various antibiotic classes and therefore able to survive in hospital environment and causing often nosocomial infections [7-8, 12].

A recent study reveals the high incidence of antibiotic resistance to three pathogens in five African countries Algeria, Egypt, Morocco, Senegal and Tunisia. Antibiotic resistance in these countries has reached alarming proportions to Pseudomonas aeruginosa including resistance to the two most valuable drugs ceftazidime and imipenem (Table 1)

Table 1. Antibiotic susceptibility for 414 strains of Pseudomonas aeruginosa from Morocco and neighboring countries.

\begin{tabular}{|c|c|c|c|c|c|c|c|c|c|c|c|c|c|}
\hline \multirow{3}{*}{ Antibiotic } & \multirow{3}{*}{$\begin{array}{c}\text { Interpretation } \\
\mathrm{mg} / \mathrm{l}\end{array}$} & \multicolumn{12}{|c|}{ Country } \\
\hline & & \multicolumn{2}{|c|}{ Algeria } & \multicolumn{2}{|c|}{ Egypt } & \multicolumn{2}{|c|}{ Morocco } & \multicolumn{2}{|c|}{ Senegal } & \multicolumn{2}{|c|}{ Tunisia } & \multicolumn{2}{|c|}{ Total } \\
\hline & & $n=60$ & $\%$ & $n=56$ & $\%$ & $n=120$ & $\%$ & $n=58$ & $\%$ & $n=120$ & $\%$ & $n=414$ & $\%$ \\
\hline \multirow{3}{*}{ Levofloxacin } & $S \leqslant 1$ & 54 & 89.8 & 28 & 50.0 & 66 & 55.1 & 52 & 89.7 & 88 & 73.3 & 288 & 69.6 \\
\hline & I & 0 & 0 & 2 & 3.6 & 1 & 0.8 & 1 & 1.7 & 2 & 1.7 & 6 & 1.5 \\
\hline & $R>2$ & 6 & 10.2 & 26 & 46.4 & 53 & 44.1 & 5 & 8.6 & 30 & 25.0 & 120 & 28.9 \\
\hline \multirow{3}{*}{ Ciprofloxacin } & $S \leqslant 0.5$ & 54 & 89.8 & 29 & 51.8 & 67 & 55.9 & 53 & 91.4 & 88 & 73.3 & 291 & 70.1 \\
\hline & 1 & 1 & 1.7 & 0 & 0 & 1 & 0.8 & 0 & 0 & 4 & 3.3 & 6 & 1.5 \\
\hline & $R>1$ & 5 & 8.5 & 27 & 48.2 & 52 & 43.2 & 5 & 8.6 & 28 & 23.3 & 117 & 28.4 \\
\hline \multirow{2}{*}{ Colistin } & $S \leqslant 4$ & 60 & 100.0 & N/A & 120 & 100.0 & 58 & 100.0 & 120 & 100.0 & 358 & 100.0 & 87.50 \\
\hline & $R>4$ & 0 & 0 & N/A & 0 & 0 & 0 & 0 & 0 & 0 & 0 & 0 & 62.50 \\
\hline \multirow{2}{*}{ Ceftazidime } & $S \leqslant 8$ & 50 & 83.1 & 38 & 68.4 & 86 & 72.0 & 57 & 98.3 & 103 & 85.8 & 334 & 80.8 \\
\hline & $R>8$ & 10 & 16.9 & 18 & 31.6 & 34 & 28.0 & 1 & 1.7 & 17 & 14.2 & 80 & 19.2 \\
\hline \multirow{3}{*}{ Imipenem } & $S \leqslant 4$ & 54 & 89.8 & 40 & 71.9 & 91 & 75.4 & 58 & 100.0 & 97 & 80.8 & 340 & 82.1 \\
\hline & I & 1 & 1.7 & 7 & 12.3 & 3 & 2.5 & 0 & 0 & 4 & 3.3 & 15 & 3.6 \\
\hline & $R>8$ & 5 & 8.5 & 9 & 15.8 & 26 & 22.0 & 0 & 0 & 19 & 15.8 & 59 & 14.3 \\
\hline \multirow{2}{*}{ Tobramycin } & $S \leqslant 4$ & 60 & 100.0 & 35 & 63.2 & 83 & 69.5 & 54 & 93.1 & 100 & 83.3 & 332 & 80.3 \\
\hline & $R>4$ & 0 & 0 & 21 & 36.8 & 37 & 30.5 & 4 & 6.9 & 20 & 16.7 & 82 & 19.7 \\
\hline
\end{tabular}


Vol. 10 No. 1:5

doi: $10.3823 / 843$
[20]. In addition, the study documented high rates of Streptococcus pneumonia penicillin-resistant in Algeria, Egypt, Morocco and Senegal (Table 2), and methicillin-resistant $S$. aureus (MRSA) in Alge- ria and Egypt, Morocco, Senegal and Tunisia with the emergence of Staphylococcus strains intermediate resistant to teicoplanin. (Table 3). There are also numerous recent studies from Algeria, Moroc-

Table 2. Antibiotic susceptibility for 218 strains of Streptococcus pneumonia. from Morocco and neighboring countries.

\begin{tabular}{|c|c|c|c|c|c|c|c|c|c|c|c|}
\hline \multirow{3}{*}{ Antibiotic } & \multirow{3}{*}{$\begin{array}{c}\text { Interpretation } \\
\text { mg/l }\end{array}$} & \multicolumn{10}{|c|}{ Country } \\
\hline & & \multicolumn{2}{|c|}{ Algeria } & \multicolumn{2}{|c|}{ Egypt } & \multicolumn{2}{|c|}{ Morocco } & \multicolumn{2}{|c|}{ Senegal } & \multicolumn{2}{|c|}{ Total } \\
\hline & & $n=60$ & $\%$ & $n=39$ & $\%$ & $n=101$ & $\%$ & $n=12$ & $\%$ & $n=218$ & $\%$ \\
\hline \multirow{3}{*}{ Penicillin } & $S \leqslant 0.06$ & 34 & 56.7 & 20 & 51.3 & 86 & 85.0 & 11 & 91.7 & 152 & 69.7 \\
\hline & I & 25 & 41.7 & 16 & 41.0 & 15 & 15.0 & 1 & 8.3 & 62 & 28.4 \\
\hline & $R>2$ & 1 & 1.7 & 3 & 7.7 & 0 & 0 & 0 & 0 & 4 & 1.8 \\
\hline \multirow{3}{*}{ Amoxicillin } & $S \leqslant 0.5$ & 51 & 85.0 & 31 & 79.5 & 95 & 94.0 & 12 & 100.0 & 192 & 88.0 \\
\hline & I & 9 & 15.0 & 6 & 15.4 & 6 & 6.0 & 0 & 0 & 23 & 10.6 \\
\hline & $R>2$ & 0 & 0 & 2 & 5.1 & 0 & 0 & 0 & 0 & 3 & 1.4 \\
\hline \multirow{3}{*}{ Cefotaxime } & $S \leqslant 0.5$ & 57 & 95.0 & 29 & 74.3 & 98 & 97.0 & 12 & 100.0 & 202 & 92.6 \\
\hline & I & 3 & 5.0 & 9 & 23.1 & 3 & 3.0 & 0 & 0 & 15 & 6.9 \\
\hline & $R>2$ & 0 & 0 & 1 & 2.6 & 0 & 0 & 0 & 0 & 1 & 0.5 \\
\hline \multirow{3}{*}{ Cefpodoxime } & $S \leqslant 0.25$ & 42 & 70.0 & 28 & 71.8 & 93 & 91.8 & 11 & 91.7 & 176 & 80.9 \\
\hline & I & 3 & 5.0 & 2 & 5.1 & 4 & 4.1 & 0 & 0 & 9 & 4.2 \\
\hline & $R>0.5$ & 15 & 25.0 & 9 & 23.1 & 4 & 4.1 & 1 & 8.3 & 33 & 14.9 \\
\hline \multirow{3}{*}{ Erythromycin } & $S \leqslant 0.25$ & 37 & 61.7 & 24 & 61.5 & 86 & 85.1 & 12 & 100.0 & 159 & 72.9 \\
\hline & I & 0 & 0 & 0 & 0 & 0 & 0 & 0 & 0 & 1 & 0.5 \\
\hline & $R>0.5$ & 23 & 38.3 & 15 & 38.5 & 15 & 14.9 & 0 & 0 & 58 & 26.6 \\
\hline \multicolumn{2}{|c|}{ PNSP + erythromycin R } & 18 & 30.0 & 11 & 28.2 & 4 & 3.9 & 0 & 0 & 37 & 16.9 \\
\hline
\end{tabular}

Table 3. Antibiotic susceptibility for 428 strains of Staphylococcus aureus from Morocco and neighboring countries.

\begin{tabular}{|c|c|c|c|c|c|c|c|c|c|c|c|c|c|}
\hline \multirow{3}{*}{ Method } & \multirow{3}{*}{$\begin{array}{l}\text { Antibiotic and } \\
\text { interpretation }\end{array}$} & \multicolumn{12}{|c|}{ Country } \\
\hline & & \multicolumn{2}{|c|}{ Algeria } & \multicolumn{2}{|c|}{ Egypt } & \multicolumn{2}{|c|}{ Morocco } & \multicolumn{2}{|c|}{ Senegal } & \multicolumn{2}{|c|}{ Tunisia } & \multicolumn{2}{|c|}{ Total } \\
\hline & & $n=72$ & $\%$ & $n=59$ & $\%$ & $n=115$ & $\%$ & $n=60$ & $\%$ & $n=122$ & $\%$ & $n=428$ & $\%$ \\
\hline $\begin{array}{l}\text { Oxacillin + } \\
\text { cefoxitin } \\
\text { disc }\end{array}$ & Methicillin $\mathrm{R}$ & 33 & 45.8 & 37 & 62.7 & 13 & 11.3 & 3 & 5.0 & 22 & 18.0 & 108 & 25.2 \\
\hline \multirow{2}{*}{ MIC Etest } & Teicoplanin $S \leqslant 2$ mg/l & 72 & 100.0 & 45 & 76.3 & 108 & 93.9 & 60 & 100.0 & 118 & 96.7 & 403 & 94.2 \\
\hline & Teicoplanin R>2 mg/l & 0 & 0 & 14 & 23.7 & 7 & 6.1 & 0 & 0 & 4 & 3.3 & 25 & 5.8 \\
\hline \multirow{3}{*}{ MIC Etest } & Levofloxacin $\mathrm{S} \leqslant 1 \mathrm{mg} / \mathrm{l}$ & 64 & 88.9 & 51 & 86.4 & 107 & 93.0 & 60 & 100.0 & 111 & 91.0 & 393 & 91.8 \\
\hline & Levofloxacin I & 6 & 8.3 & 1 & 1.7 & 1 & 0.9 & 0 & 0 & 1 & 0.8 & 9 & 2.1 \\
\hline & Levofloxacin R>2 mg/l & 2 & 2.8 & 7 & 11.9 & 7 & 6.1 & 0 & 0 & 10 & 8.2 & 26 & 6.1 \\
\hline Disc & Fosfomycin S & 72 & 100.0 & 59 & 100.0 & 115 & 100.0 & 60 & 100.0 & 119 & 97.5 & 425 & 99.3 \\
\hline Disc & Erythromycin S & 54 & 75.0 & 23 & 39.0 & 102 & 88.7 & 57 & 95.0 & 99 & 81.1 & 335 & 78.3 \\
\hline Disc & Gentamicin S & 62 & 86.2 & 17 & 29.2 & 110 & 95.7 & 60 & 100.0 & 117 & 95.9 & 366 & 85.5 \\
\hline Disc & Pristinamycin S & 67 & 93.1 & 40 & 67.8 & 114 & 99.1 & 60 & 100.0 & 122 & 100.0 & 403 & 94.2 \\
\hline
\end{tabular}


co, Libya and Tunis which have reported the wide spread of multidrug resistance and extended-spectrum $\beta$-lactamases genes among Gram-negative bacteria such as Enterobacteriaceae, particularly among E. coli and Klebsiella pneumoniae isolates from patients [21-28]. These findings are alarming and should encourage to implement urgently the recommendations of the WHO to employ regular monitoring and surveillance of bacterial resistance to antibiotics in these countries [3]. In addition, a new study from Morocco has reported on the emergence of carbapenemases genes, such as bla OXA-24, bla OXA-23, bla OXA-58 and bla NDM in A. baumannii isolates from three hospitals [29]. Another study also reported by Uwingabiye on the production of carbapenemase in $A$. baumannii isolates, collected from different patients and different places of a Moroccan hospital [30]. The emergence and spread of carbapenemase-producing carbapenem-resistant bacteria is a significant clinical and public health concern since it is associated with high mortality [31].

\section{Dangerous consequence of bacterial resistance}

What is of most concern is that common bacterial infections such as streptococcal throat may once again become untreatable due to develop of bacterial resistance. The $\mathrm{WHO}$ has outlined what a possible future could look like if antibiotic resistance grows with higher speed. They have named it the "post-antibiotic world". This world looks a lot less like Pasteur's hope of the future destruction of disease and much more like the pre-penicillin era when infectious disease dominated. The WHO warns that antibiotic resistance may soon result in simple infections becoming unmanageable and potentially even fatal $[1,3]$.

\section{Potential solutions}

Several approaches have been suggested by scientists, physicians, and other professionals for the con- trol of further drug resistance development. These include educating the public about the proper use of antibiotics and the consequences of misusing them, holding physicians and health care professionals to a much higher standard when it comes to diagnosing and treating patients with bacterial illness. Additionally, appling stricter regulations for when antibiotics can and should be prescribed, and increasing the search for discovering of new antibiotics. Multifaceted interventions that attack the problem from several sides will be the most effective approach in fighting back against antibiotic resistance. The interventions that have shown the best results in countries worldwide include: the enforcement of policies prohibiting the sale and purchase of antibiotics without a valid prescription from a qualified dispensing physician or certain cases of pharmacist. Second, the use of stewardship programs explaining the importance of the proper use of antibiotics to the public, the enforcement of laboratory culture testing before prescribing antibiotics, teaching physicians how to better manage patient treatment, and putting incentives in place for physicians who adhere to these guidelines and regulations [7, 14]. These steps, among others, are probably necessary to slow the development of antibiotic resistance. However, we must know that antibiotic resistance once established its not easily to reverse over a short time [8].

\section{Public antibiotic education}

The public is seriously misinformed about when an antibiotic is necessary and when it is not. There is a clear need for general education about the dangerous usage of antibiotics without prescription [15]. It has been recommended to including material about antibiotics and their safe usage into public school curriculum plans. This approach could go a long way to educate the general public about the dangerous use of antibiotic without medical necessity. Informative pamphlets could be given out in government buildings such as the Social Security 
Office. Television could be particularly useful in this aspect. Documentaries and news stories could go a long way towards educating the public. Social media could also potentially be used as a platform in spreading the news about antibiotic resistance. Public intervention could include the publication of guidelines, freely offered educational sessions covering what illnesses antibiotics will and will not work for, the publication of local interviews with pharmacists, messages on radio, television, and other mass media platforms [7]. These public campaigns will have a positive effect on reducing the antibiotic crisis, but educating the public will by no means solve the entire antibiotic resistance problem [8].

\section{Physician programs and interventions}

Since bacterial infection is mostly difficult to distinguish from viral infection in respiratory tract, physicians and providers must be encouraged and even required to run rapid diagnostic tests before prescribing antibiotics to a patient [15]. This procedure will often help to show patients about the reason they are not being prescribed an antibiotic. The patients could be much less likely to put pressure on a provider to prescribe antimicrobials in situations in which they are not indicated or warranted. Culture tests are generally easily to be collected in form of a swab, blood or urine sample and sending it to the laboratory. Diagnostic testing may take a little longer and seem like an inconvenience to the patient, but the time lost from their day will pale in comparison to the impending antibiotic crisis if these measures are not taken. This is not meant to trivialize a patient's concerns, but to help them understand how antibiotics can and should be properly used for the beneficial of their health and the society as a whole. Obviously, cases such as blood sepsis and meningitis should be immediately treated with antibiotics after taking the necessary clinical samples for diagnostic testing [8].
It is highly important to educate and train physicians about proper using of antibiotics as part of a physician's medical education. It is important that physician knows how to select the correct type of antibiotics, and knows how to explain to a patient with no medical background about use of antibiotics. Once the patient is understanding that antibiotics will not reduce the duration of viral diseases, and that may be only contributing to the antibiotic resistance. The information given verbally by the provider could be backed up by informational leaflets or pamphlets that the patient can take home $[5,8]$. It is also important that doctors can set realistic expectations for patients about the duration and severity of the illnesses they are experiencing. Four days is a common running time duration for ear infections, about a week for an acute sore throat or common cold caused by a virus, two and a half weeks for a sinus infection, and up to three weeks for a severe cough or bronchitis [7]. This is of particular importance because studies have shown that many patients believe that if they suffer symptoms for more than three days, antibiotics are not only warranted but absolutely necessary [32-33].

\section{Regulations}

The antibiotics currently in use have great impact on human life, therefore they must be conserved and protected while the search continues for newer antibiotics [15]. The ultimate goal of applying regulations to proper usage of antibiotics is not simply as well as to reduce the number of antibiotics being prescribed and utilized. It is highly important to encourage the rational use of antibiotics and to ensure that they are only being prescribed to patients who need the treatment and will definitely benefit from it [7].

The Centers for Disease Control(CDC) in USA, has actually already presented a rational program to proper use of antibiotics. It is a quite aggressive plan aimed at reducing the unnecessary use of antimicrobials, so that antibiotics remain a viable 
tool for future generations [34]. In fiscal year 2016, USA congress allocated nearly $\$ 160$ million for the CDC to support different agencies and programs in the fight against antimicrobial resistance. With these investments, CDC implemented the Antibiotic Resistance Solutions Initiative, which is improving national infrastructure to detect, respond, and contain resistant infections across healthcare settings and communities. This plan does have its flaws. It is aimed primarily at hospital use of antibiotics, not towards general practitioners of family practitioners. Also, the CDC's proposed plan does not address the use of antibiotics in agriculture and livestock. The CDC includes several categories of goals in the fight against antibiotic resistance. They hope to set national goals to improve antibiotic use, particularly to cut inappropriate prescribing practices by fifty percent in hospitals and twenty percent in the general practitioner and specialists' offices. They plan to encourage effective public information and antibiotic stewardship programs, focusing their efforts on doctors' offices, hospitals, and nursing homes. The CDC has provided funding to researchers who are studying antimicrobial resistance, and those who are searching for new antimicrobial substances that could potentially be used in the treatment of patients. Interestingly, they also have begun a program in support of the early recognition of sepsis [32]. All of these initiatives sound very noble and promising. However, the CDC has yet to set forth a practical approach to fighting antimicrobial resistance. All of their planning thus far has been focused toward future initiatives, not at present time. Unfortunately, the antibiotic crisis is happening today, not in the distant future.

Pharmaceutical companies must be prevented from offering prescribing incentives to medical providers, potentially through legal action. This is probably one of the toughest issues to be overcome. It may be impossible to prevent pharmaceutical companies to offer free samples of antibiotics for physicians. For the time being, the role of "Big pharma" must be recognized and possibly countered by public pressure and governmental regulations.

Antibiotics for use in animals should be administered only by a licensed veterinarian, directly or through writing a prescription that a pharmacy can fill [15]. Antibiotics should by no means be sprayed onto crops or included in the food of livestock as a preventative measure. The link between animal health and human health must be clearly recognized and integrated into the plans taken by health professionals, regulatory agencies, and the enforcing authorities [12].

\section{Search for new antibiotics}

The search for new antibiotics is well underway, though it is moving at an alarmingly slow rate. It is much more difficult for researchers to find financial support from pharmaceutical companies to search for and develop new drugs. The time delay between the discovery of a potential new antimicrobial substance and the approval to commercially produce it is on the magnitude of decades [15]. This has led many researchers to abandon the search for new antibiotics entirely, as there is not enough interest or funding.

\section{Conclusions}

In Morocco and neighboring countries the problem of antibiotic resistance is recognized and well documented by presence of numerous published studies during recent years as shown in this review. Rapidly emerging resistant bacteria threaten the extraordinary health benefits that have been achieved until now with antibiotics. The antibiotic crisis is global, reflecting the worldwide overuse of these drugs and the lack of development of new antibiotic agents by pharmaceutical companies. Antibioticresistant infections place a substantial health and 
economic burden on the health care system and population. Coordinated efforts of all health professionals worldwide with the support of governmental regulations are needed to implement new policies, renew research efforts, and pursue steps to manage the antibiotic crisis are greatly needed.

\section{References}

1. World Health Organization (WHO). Antimicrobial resistance, cited 4 Apr 2019. https://www.who.int/news-room/fact-sheets/ detail/antimicrobial-resistance

2. Moghnieh AR, Zeina A Kanafani A Z, Tabaja ZH, Sharara LS, Awad SL, Kanj SS. Epidemiology of common resistant bacterial pathogens in the countries of the Arab League. Lancet Infect Dis 2018; (12): e379-e394.

3. WHO. Global antimicrobial resistance surveillance system (GLASS) report, 2018. http://www.who.int/glass/resources/ publications/early-implementation-report

4. Michael CA, Dominey-Howes D, Labbate M. The antimicrobial resistance crisis: causes, consequences, and management. Front public Heal. Frontiers Media SA 2014; 2: 145

5. Ackerman S, Gonzales R. The context of antibiotic overuse. Ann Intern Med 2012; 157(3):211-212

6. Arason VA, Sigurdsson AJ. The problems of antibiotic overuse. Scand J Prim Health Care 2016; 28(2): 65-66.

7. Llor C, Lars Bjerrum L Antimicrobial resistance: Risk associated with antibiotic overuse and initiatives to reduce the problem. Ther Adv Drug Sa 2014; 5(6): 229-241.

8. Levy, Stuart B, 2002. The antibiotic paradox: How the misuse of antibiotics destroys their curative Power. Cambridge, MA: Perseus Publication, USA.

9. Hhm H, Ma M, Jin G, Jin Y, Khalifa I, Zeng Q, et al. Virulence factors of staphylococcus aureus isolated from korean pork bulgogi: Enterotoxin production and antimicrobial resistance. Korean J Food Sci An 2015; 35(4): 502-506.

10. Lashley, R. Felissa and Jerry D. Durham,2002. Emerging infectious diseases: Trends and issues. New York: Springer Publication, USA.

11. Schmidt, Michael A, 2009. Beyond antibiotics: Strategies for living in a world of emerging infections and antibiotic-resistant bacteria. Berkeley, CA: North Atlantic Books,USA.

12. Shallcross, Laura J, and Dame Sally C Davies. (2014). Antibiotic overuse: A key driver of antimicrobial resistance. Brit J Gen Pract 2014; 64(629): 604-605.

13. Huttner B, Harbarth S. Antibiotics are not automatic anymore' the French national campaign to cut antibiotic overuse. PLoS Medicine 2009; 6(6: e1000080.
14. Mainous AG 3rd, Hueston WJ, Love MM, Evans ME, Finger R. (2000). An evaluation of statewide strategies to reduce antibiotic coveruse. Fam Med 2000; 32(1): 22-915.

15. McKenna, John, 2014. Anti-Biotics: Are they curing us or killing us? Dublin: Gill and McMillan, England

16. Shallcross LJ, Dame SC D. Antibiotic overuse: A key driver of antimicrobial resistance. Br J Gen Pract; 2014; 64(629): 604-605.

17. Sahreena L, Kunyan Z. Methicillin-Resistant Staphylococcus aureus: Molecular Characterization, Evolution, and Epidemiology. Clin Microbiol Rev 2018; 31 (4).

18. Foster TJ. Antibiotic resistance in Staphylococcus aureus. Current status and future prospects. FEMS Microbiol Rev. 2017; 1; 41(3):430-449.

19. Holger S, Breitrück A. Clostridium difficile - From Colonization to Infection. Front. Microbiol 2018; https://doi.org/10.3389/ fmicb.2018.00646.

20. Zerouali K, Ramdani-Bouguessa N, Boye C, Hammami A\& The Active 2 Group. Multicentric study in five African countries of antibiotic susceptibility for three main pathogens: Streptococcus pneumoniae, Staphylococcus aureus, and Pseudomonas aeruginosa, J Chemother 2016; 28: 266-272.

21. Zorgani A, Almagatef A, Sufya N, Bashein A, Tubbal A. Detection of CTX-M-15 Among Uropathogenic Escherichia coli Isolated from Five Major Hospitals in Tripoli, Libya. Oman Med J. 2017; 32(4): 322-327.

22. Mathlouthi N, Al-Bayssari C, El Salabi A, Bakour S, Ben Gwierif $S$, Zorgani AA, Jridi Y, Ben Slama K, Rolain JM, Chouchani C. Carbapenemases and extended-spectrum $\beta$-lactamases producing Enterobacteriaceae isolated from Tunisian and Libyan hospitals. J Infect Dev Ctries. 2016; 2; 10(7):718-27.

23. Yahiaoui M, Robin F, Bakour R, Hamidi M, Bonnet R, Messai $Y$. Antibiotic resistance, virulence, and genetic background of community-acquired uropathogenic Escherichia coli from Algeria. Microb Drug Resist 2015; 21: 516-26.

24. Abujnah A, Zorgani A, Sabri M, El-Mohammady $H$, Khalek R, Ghenghesh K. Multidrug resistance and extended-spectrum $\beta$-lactamases genes among Escherichia coli from patients with urinary tract infections in northwestern Libya. Libyan J Med 2015; 10: 26412.

25. Mansour W, Grami R, Ben Haj Khalifa A, et al. Dissemination of multidrug-resistant blaCTX-M-15/IncFllk plasmids in Klebsiella pneumoniae isolates from hospital- and community-acquired human infections in Tunisia. Diagn Microbiol Infect Dis 2015; 83: 298-304.

26. Alem N, Frikh M, Srifi A, et al. Evaluation of antimicrobial susceptibility of Escherichia coli strains isolated in Rabat University Hospital (Morocco). BMC Res Notes 2015; 8: 392.

27. Arhoune B, Oumokhtar B, Hmami $F$, et al. Rectal carriage of extended-spectrum $\beta$-lactamase- and carbapenemaseproducing Enterobacteriaceae among hospitalised neonates in a neonatal intensive care unit in Fez, Morocco. J Glob Antimicrob Resist 2017; 8: 90-96. 
28. Barguigua A, El Otmani F, Talmi $M$, et al. Prevalence and genotypic analysis of plasmid-mediated $\beta$-lactamases among urinary Klebsiella pneumoniae isolates in Moroccan community. J Antibiot 2013; 66: 11-16.

29. Hanane El Hafa, Kawtar Nayme, Najia El Hamzaoui, Itto Maroui, Mohammed Sbiti, Khalid Zerouali K. Dissemination of carbapenem-resistant Acinetobacter baumannii strains carrying the blaGES, blaNDM and blaOXA23 in Morocco. Germs. 2019; 9(3): 133-141.

30. Natoubi S, Barguigua A, Zerhouni N, et al. First report of an OXA-58 carbapenemase producing Acinetobacter baumannii isolated from urinary tract infection in Morocco. Afr J Urol. 2017; 23:66-7.

31. Bonomo AR, Burd ME, Conly J, Limbago MB, Poirel L, Segre AJ, et al. Carbapenemase-Producing Organisms: A Global Scourge Clin Infect Dis. 2018; 66(8): 1290-1297.

32. Elias C, Moja L, Mertz D, et al. Guideline recommendations and antimicrobial resistance: the need for a change. BMJ Open 2017; 7: e016264.

33. Battin, M. Pabst. (2009). The patient as victim and vector: Ethics and infectious disease. New York: Oxford UP,USA.

34. CDC. Antibiotic Use in the United States, 2017: Progress and Opportunities. Atlanta, GA: US Department of Health and Human Services, CDC.

\author{
doi: $10.3823 / 843$
}

